\title{
Papel potencial do enfermeiro no enfrentamento do problema da tuberculose junto ao Agente Comunitário de Saúde no Programa de Controle da Tuberculose
}

Recebido em 09/04/2012

Aprovado em: 27/05/2013
Alaine Maria da Costa ${ }^{1}$

Gislane de Sousa Rodrigues ${ }^{2}$

Tatiana Maria de Melo Guimarães dos Santos ${ }^{3}$

Resumo: Objetivou-se refletir e discutir sobre o papel potencial do enfermeiro no enfrentamento do problema da tuberculose junto ao Agente Comunitário de Saúde no Programa de Controle da Tuberculose. Trata-se de pesquisa descritiva, de abordagem quantitativa, com desenho de estudo transversal. A amostra constituiu-se de 121 Agentes Comunitários de Saúde (ACS) da Estratégia Saúde da Família (ESF) - zona urbana de Teresina. Evidenciou-se que $71,1 \%$ dos sujeitos admitiram procurar o enfermeiro da ESF em caso de dúvidas frente a casos suspeitos de tuberculose. Ao enfermeiro cabem intervenções pertinentes no controle da tuberculose tanto no âmbito político quanto operativo.

Palavras-Chave: Enfermagem, Atenção Básica à Saúde, Tuberculose.

\section{The potential role of nurses in addressing the problem of tuberculosis with the Community Health Agent Program Control Tuberculosis}

Abstract: The objective of this study was to reflect and discuss about the potential role of the nurses at the tuberculosis combat, helping the Community Health Agent at the Program of Tuberculosis Control. This is a descriptive study that uses a quantitative approach, with a cross-sectional study design. The group studied was formed of 121 Community Health Agents of The Family Health Strategy in the urban zone of Teresina. It was observed that $71.1 \%$ of the agents admitted to need help of a nurse when they have doubts related to suspected cases of tuberculosis. Relevant interventions in tuberculosis control are responsibility of the nurses at a political and an operational scope.

Keywords: Nursing, Basic Attention Health, Tuberculosis.

El papel potencial de las enfermeras para resolver el problema de la tuberculosis con el control de la Salud de la Comunidad del Agente de Program Tuberculosis. Resumen: Se objetivó con este estudio reflexionar y debatir sobre el papel potencial de los enfermeros en la lucha contra la tuberculosis, junto a los agentes comunitarios de salud en el Programa de Control de la Tuberculosis. Este es un estudio descriptivo que utiliza un abordaje cuantitativo, con un diseño de estudio transversal. El grupo estudiado poseía 121 ACS de la Estrategia Salud de la Familia - zona urbana de Teresina. Se observó que $71,1 \%$ de los agentes admiten buscar a un enfermero cuando existen dudas en relación a los casos sospechosos de tuberculosis. Al enfermero, caben las intervenciones pertinentes en el control de la tuberculosis tanto en el alcance político como operacional

Palabras Clave: Enfermería, Atención Básica a la Salud, Tuberculosis.

\section{INTRODUÇÃO}

onsiderada pela Organização Mundial de Saúde (OMS) como preocupação sanitária internacional, a tuberculose (TB), apesar de ser uma doença que acompanha o homem desde as civilizações mais antigas, continua a merecer especial atenção dos profissionais de saúde e da sociedade como um todo, pois ainda obedece a todos os critérios de priorização de um agravo em saúde pública, a saber, grande magnitude, transcendência e vulnerabilidade ${ }^{(1)}$. Mesmo após a descoberta da terapêutica eficaz e da disponibilidade de muitas tecnologias de saúde do mundo moderno ao alcance, a tuberculose permanece notória no cenário epidemiológico mundial ${ }^{(2)}$.

Esta patologia atinge grandes proporções em função da via de transmissão, está vinculada ao perfil socioeconômico da população, e é severamente estigmatizada, razões estas que contribuem generosamente para o agravo da situação, podendo causar ruptura ou modificações expressivas na reprodução social do indivíduo diante da sociedade.
No cuidado à tuberculose, a Estratégia Saúde da Família (ESF) visa à obtenção precoce do diagnóstico, uma das determinações do Programa Nacional de Controle da Tuberculose (PNCT). Nas visitas domiciliares almeja-se a detecção de casos sintomáticos respiratórios (SR) e contatos, principalmente, de casos bacilíferos e crianças ${ }^{(4)}$.

Para alcançar as metas propostas no programa, o Ministério da Saúde (MS) propôs a concretização do Programa de Agentes Comunitários de Saúde (PACS) e da ESF remodelando a Atenção Básica. Dessa forma, as ações de vigilância em saúde podem proporcionar melhora no acompanhamento de casos, e conseqüente diminuição dos índices de abandono, aumentando as taxas de cura, contribuindo assim para o controle efetivo da $\mathrm{TB}^{(5)}$.

O enfermeiro é um importante membro da equipe básica multidisciplinar, o que eleva o nível de crescimento e reconhecimento social deste profissional, é por ser ele um componente ativo no processo de consolidação da ESF como política integrativa e humanizadora da saúde. O que amplia

'Acadêmica do $8 \circ$ Período do Curso de Enfermagem da Faculdade Santo Agostinho - FSA. Email: alainemaria@bol.com.br

${ }^{2}$ Acadêmica do $8^{\circ}$ Período do Curso de Enfermagem da Faculdade Santo Agostinho - FSA. Email:girodrigueslima@hotmail.com

${ }^{3}$ Enfermeira. Mestre em Enfermagem pela Universidade Federal do Piauí - UFPI. Docente do Curso de Enfermagem da Faculdade Santo Agostinho - FSA. Email:

tatianaenfermeira@yahoo.com.br 
a visibilidade e os olhares sobre a prática destes profissionais, evidenciado pelo aumento do número de estudos que abordam este tema ${ }^{(6)}$.

E apesar das dificuldades referidas pelos enfermeiros da ESF, consideram-nas propulsoras de mudanças que, contribuirão para o fortalecimento e avanço do SUS. Algumas responsabilidades dos enfermeiros deverão ocorrer de modo a favorecer a compreensão da realidade em todas as suas dimensões, levando à construção de uma nova prática em saúde, mais integral, humanista e disposta a romper com as ações do modelo "queixas-condutas", à medida que estará definindo o novo papel nesta também nova perspectiva ${ }^{(6)}$.

$O$ enfermeiro tem a oportunidade de exercer papel fundamental no cuidado ao paciente com tuberculose e seus familiares durante o cuidado domiciliar e comunitário, cabendo-lhe educá-los sobre métodos de controle da infecção(7). Como educador, esse profissional ajuda a prevenir ou atenuar maiores danos que possam acometer o portador da TB e seus contatos, explicitando a relevância do cumprimento do tratamento bem como estimulando à adesão a terapêutica ${ }^{(8)}$. Além disto, tem papel essencial junto à equipe que supervisiona, a saber: Auxiliares e Técnicos de Enfermagem e ACS, sendo responsável pela orientação e capacitação destes.

Compete lhe converter o ato de cuidar e educar com base no senso comum, tornando-o mais coerente, científico e conscientizando-se de sua realidade concreta em relação ao sistema de saúde instituído(9). Para tanto, deve fazer uso das práticas de educação em saúde, que se configuram em recursos por meio dos quais o conhecimento científico produzido orienta ações intermediadas pelos profissionais de saúde que atingem a vida cotidiana das pessoas, na medida em que compreendem os condicionantes do processo saúde-doença, os quais oferecem subsídios para a adoção de hábitos e condutas saudáveis que, como tais, contribuem para a melhoria da qualidade de vida ${ }^{(10)}$

Por isso, o MS julga ser imprescindível investimento na educação em saúde, devendo ser empregado pelas equipes da ESF, enfatizando as práticas de educação e promoção da saúde, trabalhar os conteúdos de forma contextualizada e, assim, estimular os profissionais da área a se tornarem atuantes, participativos, autônomos e críticos ${ }^{(11)}$.

Todos os planos nacionais e consensos para o controle da tuberculose enfatizaram a sua integração à atenção básica, utilizando-se da ESF como forma de ampliar o acesso ao diagnóstico e ao tratamento da tuberculose em todo o Brasil seguindo a diretriz do PNCT de "horizontalização" do combate à tuberculose, por meio da expansão de suas atividades para todos os serviços de saúde do SUS ${ }^{(12)}$.

\section{OBJETIVO}

Levando em consideração o ACS, alvo da nossa investigação na pesquisa original, e seu papel no cenário da Saúde Pública, justifica-se o trabalho, vinculando a atuação deste à do enfermeiro da ESF a quem cabe a missão de coordenar, realizar atividades de educação permanente, capacitar e supervisioná-lo. Assim, o objetivo desse artigo foi refletir e discutir sobre o papel potencial do enfermeiro no enfrentamento do problema da tuberculose junto ao ACS no Programa de Controle da Tuberculose.

\section{METODOLOGIA}

Este artigo constitui um recorte originado da pesquisa desenvolvida para conclusão do curso Bacharelado em Enfermagem. $\mathrm{O}$ projeto maior, do qual essa pesquisa faz parte, intitula-se: "Conhecimento e atuação do Agente Comunitário de Saúde no Programa de Controle da Tuberculose". Trata-se de uma pesquisa de campo descritiva, de abordagem quantitativa, que utilizou o desenho de estudo transversal.

Desenvolveu-se o estudo em Unidades de Saúde da Família, da Regional Sul de Saúde da cidade de Teresina-PI, composta por 80 equipes, sendo uma PACS, e cinco na zona rural. Realizou-se a pesquisa apenas nas ESF localizadas em zona urbana. Participaram do estudo 121 ACS, com faixa etária entre 21 e 63 anos, predominantemente mulheres (74,4\%).

Os dados foram coletados por meio da aplicação de formulário estruturado com perguntas abertas e fechadas, aplicado de forma presencial pelas pesquisadoras, sendo avaliada a precisão das respostas.

A princípio, os questionários foram digitados no programa Microsoft Excel e, importados para o programa Statistical Package for the SocialSciences - SPSS (versão 12.0 for Windows), e, em seguida, tabulados e analisados estatisticamente.

Respeitou-se o que preconiza a Resolução 196/96(13), sobre Diretrizes e Normas Regulamentadoras do Conselho Nacional de Saúde, que trata de pesquisa com seres humanos. Os participantes assinaram um Termo de Consentimento Livre e Esclarecido. O projeto foi aprovado pela Fundação Municipal

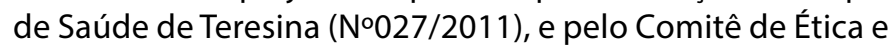
Pesquisa da Faculdade Santo Agostinho (N430/2011).

\section{RESULTADOS E DISCUSSÃO}

A ESF tem sido referenciada como tática estruturante para o alcance de mudanças significativas no contexto da Saúde Pública brasileira, sobretudo por propor relevantes mudanças na forma de conduzir o trabalho em saúde e apresenta potencialidades em contribuir na construção de um novo paradigma assistencial voltado para a prática humanizadora e holística(6).

De acordo com os dados obtidos nesta pesquisa, do total de 121 ACS entrevistados, em caso de dúvidas com relação a casos suspeitos de tuberculose, 5 consultam o Manual Tuberculose - Informações para o Agente Comunitário de Saúde (4,1\%), 30 procuram o médico da ESF, enquanto $71,1 \%$, ou seja, 86 deles, maioria absoluta, admitiram procurar o enfermeiro da ESF. A quantidade expressiva que se reporta ao enfermeiro nos faz constatar o que é amplamente relatado na literatura, como pode ser percebido na discussão que se segue.

No que se refere ao processo de conformação das equipes do PACS, está previsto que um profissional enfermeiro seja responsável pela supervisão dos ACS. O supervisor precisa ser tratado como elemento-chave, devendo ser a pessoa de referência para atuação do ACS, orientando suas atividades; alguém que o forma, recicla e atualiza, e tenha disponibilidade para realização destas ações ${ }^{(14)}$.

O enfermeiro desempenha papel crucial nos programas de controle, pois é parte da sua filosofia contribuir para que a pessoa, alvo da sua atenção, possa alcançar nível e qualidade de vida adequados, mais ainda, tratando-se do caso da tuberculose ${ }^{(15)}$. Não obstante, o plano regional de tuberculose 2006-2015 considera a enfermagem como um sócio histórico no trabalho contra essa enfermidade.

O MS divulga resultados onde mostram que em todas as unidades existe a presença de um profissional de saúde que responde pelas ações de controle da TB e as gerencia, e que 
$96,6 \%$ desses profissionais são enfermeiros ${ }^{(17)}$. Demonstrando que o enfermeiro exerce um papel importante na liderança e capacitação dessa equipe, contribuindo na reconstrução de uma identidade organizacional inspirada no modelo de vigilância em saúde.

Em estudo realizado no município de Vitória (ES), pesquisadores relatam que $73,5 \%$ dos enfermeiros declaram fazer supervisão dos ACS, o que remonta da concepção de cada um do que seria esta supervisão, não está restrita a presença do supervisor no campo, muito menos a ações restritivas a controle, e sim a todas as ações relacionadas à supervisão, devendo abranger desde o controle propriamente dito, até oportunizar momentos para processos de qualificação que permitam o desenvolvimento de competências que têm como eixo orientador a integralidade ${ }^{(6)}$.

Ainda, consideram ${ }^{(6)}$ a supervisão uma ferramenta de gestão de programas de saúde. O Ministério da Saúde recomenda que o enfermeiro instrutor/supervisor deva desenvolver ações específicas na coordenação e supervisão dos ACS, além das que rotineiramente desenvolvem enquanto coordenador da Unidade Básica de Saúde.

Diante deste cenário, o papel da enfermagem, categoria fundamental para guiar as atividades de saúde pública em nosso país, vem adquirindo importância especial na execução das ações de controle da tuberculose ${ }^{(1)}$. Em um estudo sobre treinamento da equipe de saúde e busca ativa na comunidade, revelou que após treinamento e sensibilização da equipe de saúde da família ocorreu aumento da detecção de casos de TB $^{(18)}$

Confirma-se, portanto, a relevância do papel do enfermeiro, enquanto líder da equipe de saúde, que deve sensibilizar, capacitar e estimular o ACS, e, por conseguinte, obterá maiores resultados na identificação precoce de casos de TB, oportunizando meios para tratamento adequado e consequentemente a quebra da cadeia de transmissão, contribuindo no alcance das metas globais no controle dessa enfermidade

Para Guerra, Sousa e Tocantins ${ }^{(19)}$, a atuação da enfermagem é para o controle da tuberculose ampla e contínua, onde o enfermeiro e sua equipe devem enfatizar a orientação desses clientes com relação à doença propriamente dita, à transmissão e prevenção da doença.
Para Paiva ${ }^{(20)}$, uma liderança diligente e bem exercida pode conseguir com que as organizações passem de um estado estático a um estado futuro, criando perspectivas e gerando potenciais, incitando nos trabalhadores a vontade de mudar e inserir novas filosofias e estratégias, mobilizando e focalizando energias e recursos existentes.

O resultado nos conduz ainda à comprovação de que o enfermeiro é figura mediadora de conhecimentos e provedora magna de cuidados, a quem cabe o papel imprescindível de treinar, orientar o ACS e equipe de saúde em geral, assim como avaliar o impacto de suas ações dentro da ESF e dentro do PCT, como os dados evidenciam.

\section{CONCLUSÃO}

Considerar os resultados aqui discutidos pode ser de grande relevância para o efetivo exercício de práticas transformadoras que precisam ser adicionadas a envolvimento e compromisso político para elaboração de políticas públicas centradas na necessidade da população.

Não há dúvidas de que a tuberculose ocorre com maior freqüência nas populações vulneráveis pela sua pobreza e condições de vida. No entanto, o mais importante não é saber se essas pessoas são pobres, é imperativo conhecer quão pobres elas são e quais as características da sua pobreza. Sendo assim o ACS, pela posição de ponte que exerce, já que compartilham uma realidade social semelhante e se encontram com maior freqüência, é o profissional de saúde com maior potencial de produção de vínculo com o doente de TB, e tradutor de suas necessidades, fornecendo subsídios ao enfermeiro nas intervenções pertinentes, pois é possível que a participação do enfermeiro ocorra desde os aspectos políticos até os operativos.

Os enfermeiros detêm em mãos uma gama imensa de atribuições e possibilidades, as quais vêm correspondendo, porém as dificuldades para a realização dessas ações idealizadas precisam ser melhor investigadas e posteriormente trabalhadas, afim de que possam se articular meios de atuar no combate e controle da Tuberculose na persecução das metas globais pleiteadas para o milênio.

\section{Referências}

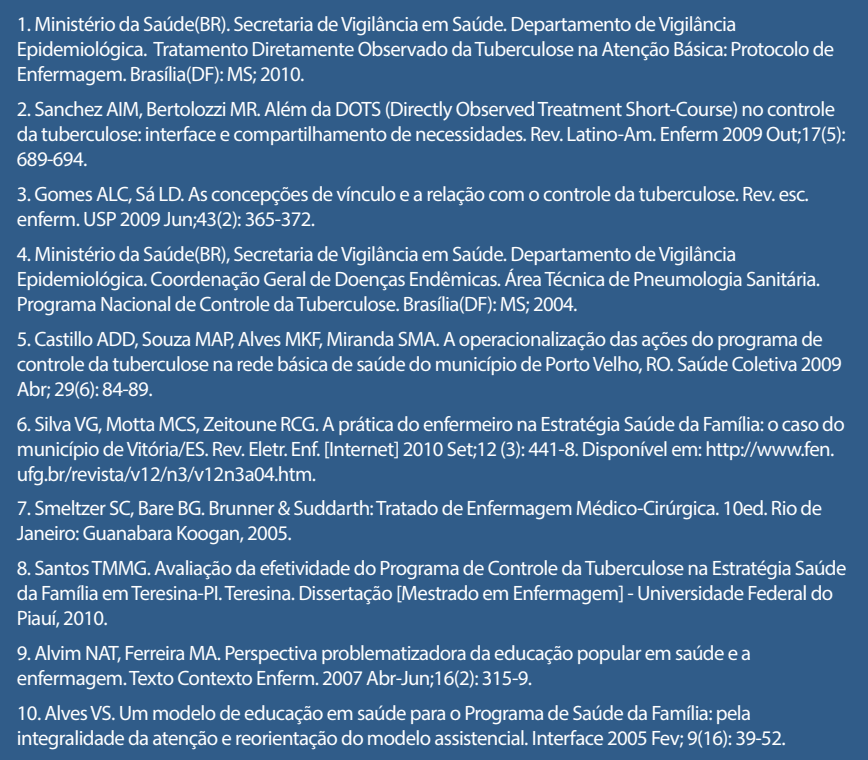

11. Alves GG, Aerts D. As práticas educativas em saúde e a Estratégia Saúde da Familia. Ciênc. saúde coletiva [online]. 2011 Jan;16(1): 319-325. Disponivel em http://www.scielosp.org/scielo.php?pid=S1413812320110001000348 script $=$ sci_arttext. 12. Maciel ELN, Vieira RCA, Milani EC, Brasil M, Fregona G, Dietze R. O agente comunitário de saúde no controle da tuberculose: conhecimentos e percepçöes. Cad. Saúde Pública 2008 Jun;24(6): 1377-1386. 13. Conselho Nacional de Saúde (Brasil). Resolução n॰ 196, de 10 outubro de 1996. Diretrizes e Normas Regulamentadoras da Pesquisa Envolvendo Seres Humanos. Diário Oficial da Uniäo 10 Out 1996; 21.082 21.085 . 14. Solla JJS, Medina MG \& Dantas MBP 1996. O PACS na Bahia: avaliaçäo do trabalho dos agentes comunitários de saúde. Saúde em Debate 1996 Jun;51:4-15. 15. Musayón Y, Loncharich N, Salazar ME, David HML, Silva I, Velásquez D. O papel da enfermagem no contro ᄀle da tuberculose uma discussão sob a perspectiva da equidade. Rev. Latino-Am. Enfermagem 2010 Jan-Fev;18(1): 130-138. 16. Organização Panamericana de Saúde. Plan Regional de Tuberculosis 2006-2015. Washington, D.C: OPS: 2006. 17. Ministério da Saúde(BR), Secretaria de Atenção Básica. Guia prático para o PSF. Brasilia(DF): MS; 2005. 18. Façanha MC, Melo MA, Vasconcelos FF, Sousa JRP, Pinheiro AS, Porto IA et al. Treinamento da equipe de saúde e busca ativa na comunidade: estratégias para a deteç̧āo de casos de TB. J Bras Pneumol. 2009 Maio;35(5): 449-454. 19. Guerra FGCM, Sousa FBAS, Tocantins FR. Cliente com tuberculose multirresistente internado em hospital geral-intensificando as açöes de enfermagem. Bol. Pneumol. Sanit. 2003 Dez; 11(2): 47-52. 20. Paiva REA. A busca de sintomáticos respiratórios de tuberculose na prática e perspectiva do Agente Comunitário de Saúde nos Distritos Sanitários de Natal. Natal. Dissertação [Mestrado em Enfermagem] Universidade Federal do Rio Grande do Norte; 2010 